\title{
ON SCATTERING PASSIVE SYSTEM NODES AND MAXIMAL SCATTERING DISSIPATIVE OPERATORS
}

\author{
OLOF J. STAFFANS
}

(Communicated by Richard Rochberg)

\begin{abstract}
There is an extensive literature on a class of linear time-invariant dynamical systems called "well-posed scattering passive systems". Such a system is generated by an operator $S$ which is called a scattering passive system node. In the existing literature such a node is typically introduced by first giving a list of assumptions which imply that $S$ is a system node and then adding an inequality which forces this system node to be scattering passive. Here we proceed in the opposite direction: we start by requiring that $S$ satisfies the passivity inequality and then ask the question, what additional conditions are needed in order for $S$ to be a system node? The answer is surprisingly simple: A necessary and sufficient condition for an operator $S$ to be a scattering passive system node is that $S$ is closed and maximal within the class of operators that satisfy the passivity inequality. In the absence of external inputs and outputs, this condition is identical to the standard condition which characterizes the class of operators which generate contraction semigroups on Hilbert spaces.
\end{abstract}

\section{INTRODUCTION}

There is an extensive literature on a class of linear time-invariant dynamical systems called "well-posed scattering passive systems"; see, e.g., AN96, MS06, MSW06, Sta01, Sta02a, Sta02b, Sta05, SW12, WST01. Such a system has an input space $\mathcal{U}$, a state space $\mathcal{X}$, and an output space $\mathcal{Y}$, all of which are Hilbert spaces, and it is generated by a closed operator $S:\left[\begin{array}{l}\mathcal{X} \\ \mathcal{U}\end{array}\right] \rightarrow\left[\begin{array}{l}\mathcal{X} \\ \mathcal{Y}\end{array}\right]$ with dense domain. Classical trajectories of the system on the time interval $\mathbb{R}^{+}:=[0, \infty)$ consist of triples of functions $(u, x, y)$, where $u \in C\left(\mathbb{R}^{+} ; \mathcal{U}\right), x \in C^{1}\left(\mathbb{R}^{+} ; \mathcal{X}\right)$, and $y \in C\left(\mathbb{R}^{+} ; \mathcal{Y}\right)$ satisfying

$$
\left[\begin{array}{l}
\dot{x}(t) \\
y(t)
\end{array}\right]=S\left[\begin{array}{l}
x(t) \\
u(t)
\end{array}\right], \quad t \in \mathbb{R}^{+} .
$$

The operator $S$ is usually supposed to be (at least) a system node, i.e., to satisfy the following conditions:

Definition 1.1. By a system node on a triple of Hilbert spaces $(\mathcal{X}, \mathcal{U}, \mathcal{Y})$ we mean a linear operator $S:\left[\begin{array}{l}\mathcal{X} \\ \mathcal{U}\end{array}\right] \rightarrow\left[\begin{array}{l}\mathcal{X} \\ \mathcal{Y}\end{array}\right]$ with the following properties. We denote $\operatorname{dom}(A)=$ $\left\{x \in \mathcal{X} \mid\left[\begin{array}{l}x \\ 0\end{array}\right] \in \operatorname{dom}(S)\right\}$, define $A: \operatorname{dom}(A) \rightarrow \mathcal{X}$ by $A x=P_{\mathcal{X}} S\left[\begin{array}{l}x \\ 0\end{array}\right]$ (where $P_{\mathcal{X}}$ is the projection of $\left[\begin{array}{l}\mathcal{X} \\ \mathcal{Y}\end{array}\right]$ onto $\mathcal{X}$ ), and require the following conditions to hold:

Received by the editors August 20, 2011.

2010 Mathematics Subject Classification. Primary 47B44, 93A05, 93C25.

Key words and phrases. Scattering passive, system node, maximally dissipative operator, wellposed linear system. 
(i) $S$ is closed as an operator from $\left[\begin{array}{l}\mathcal{X} \\ \mathcal{U}\end{array}\right]$ to $\left[\begin{array}{l}\mathcal{X} \\ \mathcal{Y}\end{array}\right]$.

(ii) $P_{\mathcal{X}} S$ is closed as an operator from $\left[\begin{array}{l}\mathcal{X} \\ \mathcal{U}\end{array}\right]$ to $\mathcal{X}$ (with domain $\operatorname{dom}(S)$ ).

(iii) $A$ is the generator of a $C_{0}$ semigroup.

(iv) For every $u \in \mathcal{U}$ there exists an $x \in \mathcal{X}$ such that $\left[\begin{array}{l}x \\ u\end{array}\right] \in \operatorname{dom}(S)$.

(Definition 4.7.2 in Sta05] is more complicated, but according to Sta05, Lemma 4.7.7] it is equivalent to Definition 1.1 above.)

The assumption that $S$ is a system node implies, among others, that for a sufficiently large set of initial states $x_{0} \in \mathcal{X}$ and input functions $u$ there exist unique functions $x$ and $y$ satisfying (1.1). A system node $S$ is called passive if all the classical trajectories of (1.1) satisfy

$$
\frac{\mathrm{d}}{\mathrm{d} t}\|x(t)\|_{\mathcal{X}}^{2}+\|y(t)\|_{\mathcal{Y}}^{2} \leq\|u(t)\|_{\mathcal{U}}^{2}, \quad t \in \mathbb{R}^{+} .
$$

By integrating this inequality into

$$
\|x(t)\|_{\mathcal{X}}^{2}+\int_{0}^{t}\|y(s)\|_{\mathcal{Y}}^{2} \mathrm{~d} s \leq\|x(0)\|_{\mathcal{X}}^{2}+\int_{0}^{t}\|u(s)\|_{\mathcal{U}}^{2} \mathrm{~d} s, \quad t \in \mathbb{R}^{+},
$$

one gets still another equivalent way of characterizing scattering passivity. This integrated version is valid even for generalized trajectories of (1.1). See [Sta05, Chapter 11] for details.

It follows from (1.1) and (1.2) (together with Definition 1.1) that a system node $S$ is passive if and only if it satisfies the following condition:

$$
\text { for all }\left[\begin{array}{l}
x \\
u
\end{array}\right] \in \operatorname{dom}(S), 2 \Re\langle\dot{x}, x\rangle+\|y\|_{\mathcal{Y}}^{2} \leq\|u\|_{\mathcal{U}}^{2} \text {, where }\left[\begin{array}{l}
\dot{x} \\
y
\end{array}\right]:=S\left[\begin{array}{l}
x \\
u
\end{array}\right] .
$$

In the sequel we shall call operators satisfying condition (1.4) scattering dissipative. Note that if $\mathcal{U}=\mathcal{Y}=\{0\}$, then condition (1.4) reduces to the standard dissipativity condition on $S$, and if $\mathcal{X}=\{0\}$, then the above condition says that $S$ is a (not necessarily everywhere defined) contraction.

Here we ask the following question: Suppose that $S:\left[\begin{array}{l}\mathcal{X} \\ \mathcal{U}\end{array}\right] \rightarrow\left[\begin{array}{l}\mathcal{X} \\ \mathcal{Y}\end{array}\right]$ is a linear operator which is scattering dissipative; i.e., it satisfies (1.4). What additional conditions on $S$ do we need in order to guarantee that $S$ is a system node? The answer to this question turns out to be surprisingly simple: An operator $S$ is a scattering passive system node if and only if $S$ is closed and maximal within the class of all scattering dissipative operators $\left[\begin{array}{l}\mathcal{X} \\ \mathcal{U}\end{array}\right] \rightarrow\left[\begin{array}{l}\mathcal{X} \\ \mathcal{Y}\end{array}\right]$. This means that every closed maximal scattering dissipative operator has all the properties of a scattering passive system node (see, for example, [Sta05, Definition 4.7.2 and Lemma 4.7.3] for partial lists of these properties). Also note that, in the absense of external inputs and outputs, the above condition is identical to the standard condition which characterizes the class of operators which generate contraction semigroups on Hilbert spaces.

\section{Scattering Dissipative operators}

Throughout the rest of this article we fix three Hilbert spaces, $\mathcal{U}, \mathcal{X}$, and $\mathcal{Y}$. We denote the direct orthogonal sum of $\mathcal{X}$ and $\mathcal{U}$ by $\left[\begin{array}{l}\mathcal{X} \\ \mathcal{U}\end{array}\right]$. The identity operator in $\mathcal{X}$ is denoted by $1_{\mathcal{X}}$. The coordinate maps $\left[\begin{array}{l}x \\ u\end{array}\right] \rightarrow x$ and $\left[\begin{array}{l}x \\ u\end{array}\right] \rightarrow u$ are denoted by $\left[\begin{array}{ll}1_{\mathcal{X}} & 0\end{array}\right]$ and $\left[\begin{array}{ll}0 & 1_{\mathcal{U}}\end{array}\right]$, respectively. The map from $x \in \mathcal{X}$ to $\left[{ }_{C x}^{A x}\right] \in\left[\begin{array}{l}\mathcal{X} \\ \mathcal{U}\end{array}\right]$ is denoted by $\left[\begin{array}{l}A \\ C\end{array}\right]$. 
Definition 2.1. Let $S:\left[\begin{array}{l}\mathcal{X} \\ \mathcal{U}\end{array}\right] \rightarrow\left[\begin{array}{l}\mathcal{X} \\ \mathcal{Y}\end{array}\right]$ be a linear operator with domain $\mathcal{D}(S)$.

(i) $S$ is called scattering dissipative if it satisfies (1.4).

(ii) $S$ is called maximal scattering dissipative if, in addition, $S$ has no proper scattering dissipative extension.

Lemma 2.2. Let $S$ be scattering dissipative and define $E:\left[\begin{array}{l}\mathcal{X} \\ \mathcal{U}\end{array}\right] \rightarrow\left[\begin{array}{l}\mathcal{X} \\ \mathcal{Y}\end{array}\right]$ by

$$
E=\left[\begin{array}{cc}
\frac{1}{\sqrt{2}} 1_{\mathcal{X}} & 0 \\
0 & 1_{\mathcal{U}}
\end{array}\right]-\left[\begin{array}{r}
\frac{1}{\sqrt{2}}\left[\begin{array}{rr}
1_{\mathcal{X}} & 0
\end{array}\right] S \\
0
\end{array}\right], \quad \mathcal{D}(E)=\mathcal{D}(S) .
$$

Let $\left[\begin{array}{l}x \\ u\end{array}\right] \in \mathcal{D}(S)=\mathcal{D}(E)$, and denote $\left[\begin{array}{l}\dot{x} \\ y\end{array}\right]=S\left[\begin{array}{l}x \\ u\end{array}\right], x_{0}=\left[\begin{array}{ll}1 \mathcal{X} & 0\end{array}\right] E\left[\begin{array}{l}x \\ u\end{array}\right]$. Then

$$
2\left\|x_{0}\right\|_{\mathcal{X}}^{2}+\|u\|_{\mathcal{U}}^{2} \geq\|x\|_{\mathcal{X}}^{2}+\|\dot{x}\|_{\mathcal{X}}^{2}+\|y\|_{\mathcal{Y}}^{2} .
$$

Therefore $E$ is injective, and $E$ is closed if and only if $\mathcal{R}(E)$ is closed.

Proof. The inequality (2.2) follows from the fact that

$$
\begin{aligned}
2\left\|x_{0}\right\|^{2} & =\|x-\dot{x}\|_{\mathcal{X}}^{2}=\|x\|_{\mathcal{X}}^{2}+\|\dot{x}\|_{\mathcal{X}}^{2}-2 \Re\langle\dot{x}, x\rangle_{\mathcal{X}} \\
& \geq\|x\|_{\mathcal{X}}^{2}+\|\dot{x}\|_{\mathcal{X}}^{2}+\|y\|_{\mathcal{Y}}^{2}-\|u\|_{\mathcal{U}}^{2},
\end{aligned}
$$

where we have used the scattering dissipativity of $S$. Clearly (2.2) implies that $E$ has a bounded inverse $E^{-1}$ defined on $\mathcal{R}(E)$. In particular, $E$ is injective, and $E$ is closed if and only if $\mathcal{R}(E)$ is closed. by

Let $S$ be scattering dissipative, define $E$ as in (2.1), and define $\mathbf{T}:\left[\begin{array}{l}\mathcal{X} \\ \mathcal{U}\end{array}\right] \rightarrow\left[\begin{array}{l}\mathcal{X} \\ \mathcal{Y}\end{array}\right]$

$$
\left.\mathbf{T}=\left[\begin{array}{cc}
-1_{\mathcal{X}} & 0 \\
0 & 0
\end{array}\right]+\left(\left[\begin{array}{cc}
\sqrt{2} 1_{\mathcal{X}} & 0 \\
0 & 0
\end{array}\right]+\left[\begin{array}{ll}
0 \\
0 & 1 \mathcal{Y}
\end{array}\right] S\right]\right) E^{-1}, \quad \mathcal{D}(\mathbf{T})=\mathcal{R}(E)
$$

The easiest way to describe this transformation is to observe that if $\left[\begin{array}{l}x \\ u\end{array}\right] \in \mathcal{D}(S)$ and $S\left[\begin{array}{l}x \\ u\end{array}\right]=\left[\begin{array}{l}\dot{x} \\ y\end{array}\right]$, then $\left[\begin{array}{c}\frac{1}{\sqrt{2}}(x-\dot{x}) \\ u\end{array}\right] \in \mathcal{D}(\mathbf{T})$ and $\mathbf{T}\left[\begin{array}{c}\frac{1}{\sqrt{2}}(x-\dot{x}) \\ u\end{array}\right]=\left[\begin{array}{c}\frac{1}{\sqrt{2}}(x+\dot{x}) \\ y\end{array}\right]$; and conversely, if $\left[\begin{array}{c}x_{0} \\ u\end{array}\right] \in \mathcal{D}(\mathbf{T})$ and $\mathbf{T}\left[\begin{array}{c}x_{0} \\ u\end{array}\right]=\left[\begin{array}{c}x_{1} \\ y\end{array}\right]$, then $\left[\frac{1}{\sqrt{2}}\left(\begin{array}{c}\left.x_{0}+x_{1}\right) \\ u\end{array}\right] \in \mathcal{D}(S)\right.$ and $\mathbf{T}\left[\begin{array}{c}\frac{1}{\sqrt{2}}\left(x_{0}+x_{1}\right) \\ u\end{array}\right]=\left[\begin{array}{c}\frac{1}{\sqrt{2}}\left(x_{0}-x_{1}\right) \\ y\end{array}\right]$. In particular, these formulas show that $S$ can be recovered from $\mathbf{T}$ by the formula

$$
\begin{aligned}
& F=\left[\begin{array}{cc}
\frac{1}{\sqrt{2}} 1_{\mathcal{X}} & 0 \\
0 & 1_{\mathcal{U}}
\end{array}\right]+\left[\begin{array}{r}
\frac{1}{\sqrt{2}}\left[\begin{array}{cc}
1_{\mathcal{X}} & 0
\end{array}\right] \mathbf{T} \\
0
\end{array}\right], \quad \mathcal{D}(F)=\mathcal{D}(\mathbf{T}), \\
& S=\left[\begin{array}{cc}
1_{\mathcal{X}} & 0 \\
0 & 0
\end{array}\right]+\left(\left[\begin{array}{cc}
-\sqrt{2} 1_{\mathcal{X}} & 0 \\
0 & 0
\end{array}\right]+\left[\begin{array}{ll}
0 \\
{\left[\begin{array}{ll}
0 & 1_{\mathcal{Y}}
\end{array}\right] \mathbf{T}}
\end{array}\right]\right) F^{-1}, \quad \mathcal{D}(S)=\mathcal{R}(F),
\end{aligned}
$$

where $F=E^{-1}$. As in Sta05 and SW12, we call T the internal Cayley transform of $S$, and $S$ the inverse internal Cayley transform of $\mathbf{T}$. We remark that if $\mathcal{U}=\mathcal{Y}=$ $\{0\}$, then $E=\frac{1}{\sqrt{2}}\left(1_{\mathcal{X}}-S\right), \mathbf{T}=\left(1_{\mathcal{X}}+S\right)\left(1_{\mathcal{X}}-S\right)^{-1}$, and $S=\left(\mathbf{T}-1_{\mathcal{X}}\right)\left(\mathbf{T}+1_{\mathcal{X}}\right)^{-1}$; i.e., $\mathbf{T}$ is the standard Cayley transform of $S$. If instead $\mathcal{X}=\{0\}$, then $E=1_{\mathcal{U}}$ and $\mathbf{T}=S$.

Some of the properties of the internal Cayley transform are listed in the following theorem.

\section{Theorem 2.3.}

(i) If $S$ is scattering dissipative, then the internal Cayley transform $\mathbf{T}$ of $S$ is contractive (on its domain), and the operator $1_{\mathcal{X}}+\left[\begin{array}{cc}1_{\mathcal{X}} & 0\end{array}\right] \mathbf{T}\left[\begin{array}{c}1_{\mathcal{X}} \\ 0\end{array}\right]$ is injective. 
(ii) Conversely, if $\mathbf{T}:\left[\begin{array}{l}\mathcal{X} \\ \mathcal{U}\end{array}\right] \rightarrow\left[\begin{array}{l}\mathcal{X} \\ \mathcal{Y}\end{array}\right]$ is contractive and the operator $1_{\mathcal{X}}+$ $\left[\begin{array}{cc}1 \mathcal{X} & 0\end{array}\right] \mathbf{T}\left[\begin{array}{c}1 \mathcal{X} \\ 0\end{array}\right]$ is injective, then the inverse internal Cayley transform $S$ of $\mathbf{T}$ is well-defined through formulas (2.4), $S$ is scattering dissipative, and $\mathbf{T}$ is the internal Cayley transform of $S$.

(iii) An operator $S_{1}$ is a proper scattering dissipative extension of $S$ if and only if the internal Cayley transform $\mathbf{T}_{1}$ is a proper contractive extension of the Cayley transform $\mathbf{T}$ of $S$ with the property that $1_{\mathcal{X}}+\left[\begin{array}{ll}1_{\mathcal{X}} & 0\end{array}\right] \mathbf{T}_{1}\left[\begin{array}{c}1_{\mathcal{X}} \\ 0\end{array}\right]$ is injective.

(iv) If $S$ and $\mathbf{T}$ are related as above and $E$ is defined by (2.1), then the following conditions are equivalent:
(a) $S$ is closed;
(b) $\mathbf{T}$ is closed;
(c) $\mathcal{D}(\mathbf{T})$ is closed;
(d) E is closed;
(e) $\mathcal{R}(E)$ is closed.

Proof of (i). Let $\left[\begin{array}{l}x \\ u\end{array}\right] \in \operatorname{dom}(S)$, and let $\left[\begin{array}{l}x_{0} \\ u_{0}\end{array}\right]=E\left[\begin{array}{l}x \\ u\end{array}\right]$. Then $u_{0}=u$, and $\left[\begin{array}{l}x_{0} \\ u_{0}\end{array}\right] \in$ $\operatorname{dom}(\mathbf{T})$. Alternatively, we could have started with an arbitrary vector $\left[\begin{array}{l}x_{0} \\ u_{0}\end{array}\right] \in$ $\operatorname{dom}(\mathbf{T})$ and defined $\left[\begin{array}{l}x \\ u\end{array}\right]=E^{-1}\left[\begin{array}{l}x_{0} \\ u_{0}\end{array}\right]$, and the relationships between the vectors $x$, $u, x_{0}$, and $u_{0}$ would still be the same. Let $\left[\begin{array}{l}\dot{x} \\ y\end{array}\right]=S\left[\begin{array}{l}x \\ u\end{array}\right]$ and $\left[\begin{array}{l}x_{1} \\ y_{1}\end{array}\right]=\mathbf{T}\left[\begin{array}{l}x_{0} \\ u_{0}\end{array}\right]$. Then

$$
x_{0}=\frac{1}{\sqrt{2}}(x-\dot{x}), \quad u_{0}=u, \quad x_{0}=\frac{1}{\sqrt{2}}(x-\dot{x}), \quad y_{1}=y,
$$

and

$$
\begin{aligned}
\left\|x_{1}\right\|_{\mathcal{X}}^{2}+\|y\|_{\mathcal{Y}}^{2} & =\frac{1}{2}\|x+\dot{x}\|_{\mathcal{X}}^{2}+\|y\|_{\mathcal{Y}}^{2}=\frac{1}{2}\|x-\dot{x}\|_{\mathcal{X}}^{2}+2 \Re\langle\dot{x}, x\rangle_{\mathcal{X}}+\|y\|_{\mathcal{Y}}^{2} \\
& \leq \frac{1}{2}\|x-\dot{x}\|_{\mathcal{X}}^{2}+\|u\|_{\mathcal{U}}^{2}=\left\|x_{0}\right\|_{\mathcal{X}}^{2}+\|u\|_{\mathcal{U}}^{2},
\end{aligned}
$$

where we have used the scattering dissipativity of $S$. Thus $\mathbf{T}$ is contractive (on its domain).

To see that $1_{\mathcal{X}}+\left[\begin{array}{ll}1_{\mathcal{X}} & 0\end{array}\right] \mathbf{T}\left[\begin{array}{c}1_{\mathcal{X}} \\ 0\end{array}\right]$ is injective we take (still with the same notation) $u=0$ and $x_{1}+x_{0}=0$. Since $x_{1}+x_{0}=\frac{1}{\sqrt{2}}(x+\dot{x})+\frac{1}{\sqrt{2}}(x-\dot{x})=\sqrt{2} x$, we get $x=$ 0 , and hence $1_{\mathcal{X}}+\left[\begin{array}{ll}1_{\mathcal{X}} & 0\end{array}\right] \mathbf{T}\left[\begin{array}{c}1 \mathcal{X} \\ 0\end{array}\right]$ is injective.

Proof of (ii). It is easy to see that the operator $F$ defined in (2.4) is injective if and only if $1_{\mathcal{X}}+\left[\begin{array}{ll}1_{\mathcal{X}} & 0\end{array}\right] \mathbf{T}\left[\begin{array}{c}1_{\mathcal{X}} \\ 0\end{array}\right]$ is injective. Thus, the second half of (2.4) defines an operator $S$ on $\mathcal{R}(F)$ whenever $1_{\mathcal{X}}+\left[\begin{array}{cc}1_{\mathcal{X}} & 0\end{array}\right] \mathbf{T}\left[\begin{array}{c}1_{\mathcal{X}} \\ 0\end{array}\right]$. A computation analogous to the one above shows that $S$ is scattering dissipative, and it is easy to check that the internal Cayley transform of $S$ is $\mathbf{T}$.

Proof of (iii). This follows from (i) and (ii).

Proof of (iv). By Lemma 2.2. (d) $\Leftrightarrow$ (e). Since $\mathbf{T}$ is a contraction and $\mathcal{D}(\mathbf{T})=$ $\mathcal{R}(E)$, we have $(\mathrm{c}) \Leftrightarrow(\mathrm{e})$, and since $\mathbf{T}(\alpha)$ is a contraction we have (b) $\Leftrightarrow(\mathrm{c})$. Finally, (a) $\Leftrightarrow$ (b) since the graph of $S$ is mapped onto the graph of $\mathbf{T}$ by the boundedly invertible operator

$$
\left[\begin{array}{cccc}
\frac{1}{\sqrt{2}} 1_{\mathcal{X}} & 0 & -\frac{1}{\sqrt{2}} 1_{\mathcal{X}} & 0 \\
0 & 1_{\mathcal{U}} & 0 & 0 \\
\frac{1}{\sqrt{2}} 1_{\mathcal{X}} & 0 & \frac{1}{\sqrt{2}} 1_{\mathcal{X}} & 0 \\
0 & 0 & 0 & 1_{\mathcal{Y}}
\end{array}\right] .
$$


Theorem 2.4. If $S$ is maximal scattering dissipative, then the following conditions are equivalent:

(i) $S$ is closable.

(ii) $S$ is closed.

(iii) The domain of the internal Cayley transform $\mathbf{T}$ of $S$ is equal to $\left[\begin{array}{l}\mathcal{X} \\ \mathcal{U}\end{array}\right]$.

(iv) $\mathcal{D}(S) \cap\left[\begin{array}{l}\mathcal{X} \\ 0\end{array}\right]$ is dense in $\left[\begin{array}{l}\mathcal{X} \\ 0\end{array}\right]$.

Proof of (i) $\Leftrightarrow$ (ii). If $S$ is closable, then it is easy to see that also the closure of $S$ is a scattering dissipative operator. Thus, (i) $\Leftrightarrow$ (ii).

Proof of (ii) $\Leftrightarrow$ (iii). Clearly, if (iii) holds, then $\mathbf{T}$ is closed, and by Theorem 2.3 . also $S$ is closed. Conversely, suppose that $S$ is closed. Then by Theorem 2.3. $\mathcal{D}(\mathbf{T})=\mathcal{D}(E)$ is closed. If $\mathcal{D}(\mathbf{T}) \neq\left[\begin{array}{l}\mathcal{X} \\ \mathcal{U}\end{array}\right]$, then we can extend $\mathbf{T}$ to a contraction $\mathbf{T}_{1}$ defined on all of $\left[\begin{array}{l}\mathcal{X} \\ \mathcal{U}\end{array}\right]$ by taking $\mathbf{T}\left[\begin{array}{c}x_{0} \\ u\end{array}\right]=0$ for all $\left[\begin{array}{c}x_{0} \\ u\end{array}\right] \in \mathcal{D}(\mathbf{T})^{\perp}$. Since $1_{\mathcal{X}}+$ $\left[\begin{array}{ll}1_{\mathcal{X}} & 0\end{array}\right] \mathbf{T}\left[\begin{array}{c}1_{\mathcal{X}} \\ 0\end{array}\right]$ is injective, also $1_{\mathcal{X}}+\left[\begin{array}{ll}1_{\mathcal{X}} & 0\end{array}\right] \mathbf{T}_{1}\left[\begin{array}{c}1_{\mathcal{X}} \\ 0\end{array}\right]$ is injective. By Theorem 2.3 , the inverse internal Cayley transform $S_{1}$ of $\mathbf{T}_{1}$ is then a proper scattering dissipative extension of $S$, and consequently, $S$ cannot be maximal scattering dissipative. Thus (ii) $\Leftrightarrow$ (iii).

Proof of (iii) $\Rightarrow$ (iv). Suppose that $\mathcal{D}(\mathbf{T})=\left[\begin{array}{l}\mathcal{X} \\ \mathcal{U}\end{array}\right]$. If $\mathcal{D}(S) \cap\left[\begin{array}{l}\mathcal{X} \\ 0\end{array}\right]$ is not dense in $\left[\begin{array}{l}\mathcal{X} \\ 0\end{array}\right]$, then it follows from (2.4) that $\mathcal{R}\left(1_{\mathcal{X}}+\mathbf{A}\right)$ is not dense in $X$, where $\mathbf{A}=$ $\left[\begin{array}{cc}1_{\mathcal{X}} & 0\end{array}\right] \mathbf{T}\left[\begin{array}{c}1_{\mathcal{X}} \\ 0\end{array}\right]$. Equivalently, $1_{\mathcal{X}}+\mathbf{A}^{*}$ is not injective. Thus, there exists some nonzero $x_{0} \in X$ such that $x_{0}+\mathbf{A}^{*} x_{0}=0$, i.e., $\mathbf{A}^{*} x_{0}=-x_{0}$. Therefore

$$
\left.0=\left\|x_{0}\right\|_{\mathcal{X}}^{2}-\left\|\mathbf{A}^{*} x_{0}\right\|_{\mathcal{X}}^{2}=\left\langle x_{0}, 1_{\mathcal{X}}-\mathbf{A} \mathbf{A}^{*}\right) x_{0}\right\rangle_{\mathcal{X}}=\left\|\left(1_{\mathcal{X}}-\mathbf{A} \mathbf{A}^{*}\right)^{1 / 2} x_{0}\right\|_{\mathcal{X}}^{2} .
$$

Thus $\left(1_{\mathcal{X}}-\mathbf{A} \mathbf{A}^{*}\right)^{1 / 2} x_{0}=0$, and hence also $\left(1_{\mathcal{X}}-\mathbf{A} \mathbf{A}^{*}\right) x_{0}=0$, i.e., $\mathbf{A} \mathbf{A}^{*} x_{0}=x_{0}$. Here $\mathbf{A}^{*} x_{0}=x_{0}$, which gives $\mathbf{A} x_{0}=-x_{0}$, i.e., $x_{0}+\mathbf{A} x_{0}=0$. But this contradicts the fact that, by Theorem $2.3,1 \mathcal{X}+\mathbf{A}$ is injective. This shows that (iii) $\Rightarrow$ (iv).

Proof of (iv) $\Rightarrow$ (iii). If $\mathcal{D}(S) \cap\left[\begin{array}{l}\mathcal{X} \\ 0\end{array}\right]$ is dense in $\left[\begin{array}{c}\mathcal{X} \\ 0\end{array}\right]$, then it follows from (2.4) that $\mathcal{R}\left(1_{\mathcal{X}}+\mathbf{A}\right)$ is dense in $X$, where $\mathbf{A}=\left[\begin{array}{ll}1_{\mathcal{X}} & 0\end{array}\right] \mathbf{T}\left[\begin{array}{c}1_{\mathcal{X}} \\ 0\end{array}\right]$. Since $\mathbf{A}$ is a contraction, this implies that $1 \mathcal{X}+\mathbf{A}$ is injective, which can be seen as follows (this argument is reproduced from Phi59, p. 200]). Suppose that $x_{0}+\mathbf{A} x_{0}=0$, i.e., $\mathbf{A} x_{0}=-x_{0}$, let $x_{1} \in \mathcal{D}(\mathbf{A})$, and set $x_{2}=x_{1}+\mathbf{A} x_{1}$. Then for all $\alpha \in \mathbb{C}$,

$$
\left\|\mathbf{A}\left(x_{1}-\alpha x_{0}\right)\right\|_{\mathcal{X}}^{2} \leq\left\|x_{1}-\alpha x_{0}\right\|_{\mathcal{X}}^{2},
$$

which reduces to

$$
\alpha\left\langle x_{0}, x_{2}\right\rangle_{\mathcal{X}}+\bar{\alpha}\left\langle x_{2}, x_{0}\right\rangle_{\mathcal{X}} \leq\left\|x_{1}\right\|_{\mathcal{X}}^{2}-\left\|\mathbf{A} x_{1}\right\|_{\mathcal{X}}^{2} .
$$

Since $\alpha$ is arbitrary, it follows that $\left\langle x_{0}, x_{2}\right\rangle_{\mathcal{X}}=0$ and by assumption this must hold for a dense set of $x_{2}$. Consequently, $x_{0}=0$, and this proves that $1 \mathcal{X}+\mathbf{A}$ is injective.

If $\mathbf{T}$ is not everywhere defined, then $\mathbf{T}$ can be properly extended to an everywhere defined contraction $\mathbf{T}_{1}$. The main operator $\mathbf{A}_{1}=P_{\mathcal{X}} \mathbf{T}_{1}\left[\begin{array}{c}1_{\mathcal{X}} \\ 0\end{array}\right]$ of $\mathbf{T}$ is an extension of $\mathbf{A}$, and hence $\mathcal{R}\left(1_{\mathcal{X}}+\mathbf{A}_{1}\right)$ is dense in $\mathcal{X}$. By the same argument that we gave above, $1_{\mathcal{X}}+\mathbf{A}_{1}$ is injective. Let $S_{1}$ be the inverse internal Cayley transform of $\mathbf{T}_{1}$. Then $S_{1}$ is a proper scattering dissipative extension of $S$, which contradicts the assumption that $S$ is maximal scattering dissipative. Thus, the assumption that $\mathbf{T}$ is not everywhere defined resulted in a contradiction, and we conclude that (iv) $\Rightarrow$ (iii).

An example of a maximal dissipative operator (with $U=Y=\{0\}$ ) which does not satisfy the equivalent conditions in Theorem 2.4 is given in [Phi59, p. 201]. 
Theorem 2.5. A linear operator $S:\left[\begin{array}{l}\mathcal{X} \\ \mathcal{U}\end{array}\right] \rightarrow\left[\begin{array}{l}\mathcal{X} \\ \mathcal{Y}\end{array}\right]$ is a scattering passive system node if and only if it is closed and maximal scattering dissipative.

We remark that according to Theorem 2.4, this result remains true if we replace the requirement that $S$ is closed by the requirement that the domain of the main operator $A$ of $S$ (see Definition 1.1) is dense in $\mathcal{X}$.

Proof of Theorem 2.5. If $S$ is a scattering passive system node, then by Sta05, Theorem 11.1.5], $S$ is closed and scattering dissipative, and the internal Cayley transform $\mathbf{T}$ of $S$ is a contraction with domain $\mathcal{D}(\mathbf{T})=\left[\begin{array}{l}\mathcal{X} \\ \mathcal{U}\end{array}\right]$. Thus $\mathbf{T}$ has no proper (operator) extension, and by Theorem 2.3. $S$ does not have any proper scattering dissipative extension. Thus, $S$ is maximal scattering dissipative.

Conversely, suppose that $S$ is closed and maximal scattering dissipative. Then by Theorem 2.4, the internal Cayley transform $\mathbf{T}$ of $S$ is a contraction with domain $\mathcal{D}(\mathbf{T})=\left[\begin{array}{l}\mathcal{X} \\ \mathcal{U}\end{array}\right]$. By $[$ SW12, Proposition 4.3], $S$ is a scattering passive system node.

\section{AN EXAMPLE}

Theorem 2.5 can be used to give a very short proof of the following result (which is essentially a particular case of the main result of [SW10]).

Proposition 3.1. Let $\mathcal{U}, \mathcal{X}$, and $\mathcal{Y}$ be Hilbert spaces, let $S$ be a linear operator $\left[\begin{array}{l}\mathcal{X} \\ \mathcal{U}\end{array}\right] \rightarrow\left[\begin{array}{l}\mathcal{X} \\ \mathcal{Y}\end{array}\right]$, and let $P$ be a positive selfadjoint and boundedly invertible operator in $\mathcal{X}$. Define

$$
S_{P}=\left[\begin{array}{cc}
P & 0 \\
0 & 1_{\mathcal{Y}}
\end{array}\right] S\left[\begin{array}{cc}
P & 0 \\
0 & 1_{\mathcal{U}}
\end{array}\right], \quad \mathcal{D}\left(S_{P}\right)=\left[\begin{array}{cc}
P^{-1} & 0 \\
0 & 1_{\mathcal{U}}
\end{array}\right] \mathcal{D}(S)
$$

Then $S$ is a scattering passive system node if and only if $S_{P}$ is a scattering passive system node.

Proof. Clearly, it suffices to prove this proposition in one direction, since the other direction then follows if we interchange $S$ and $S_{P}$ and also replace $P$ by $P^{-1}$.

Suppose that $S$ is scattering passive, i.e., that $S$ is closed and maximal scattering dissipative. Let $\left[\begin{array}{c}x_{P} \\ u\end{array}\right] \in \operatorname{dom}\left(S_{P}\right)$ and denote $\left[\begin{array}{c}\dot{x}_{P} \\ y\end{array}\right]:=S_{P}\left[\begin{array}{c}x_{P} \\ u\end{array}\right]$. Let $x=P x_{P}$ and $\dot{x}=P^{-1} \dot{x}_{P}$. Then $\left[\begin{array}{l}x \\ u\end{array}\right] \in \operatorname{dom}(S)$ and $\left[\begin{array}{l}\dot{x} \\ y\end{array}\right]=S\left[\begin{array}{l}x \\ u\end{array}\right]$. Consequently,

$$
\begin{aligned}
0 & \leq 2 \Re\langle\dot{x}, x\rangle+\|y\|_{\mathcal{Y}}^{2}-\|u\|_{\mathcal{U}}^{2}=2 \Re\left\langle P^{-1} \dot{x}_{P}, P x_{P}\right\rangle+\|y\|_{\mathcal{Y}}^{2}-\|u\|_{\mathcal{U}}^{2} \\
& =2 \Re\left\langle\dot{x}_{P}, x_{P}\right\rangle+\|y\|_{\mathcal{Y}}^{2}-\|u\|_{\mathcal{U}}^{2} .
\end{aligned}
$$

This shows that $S_{P}$ is scattering dissipative. It is also easy to see that $S_{P}$ is closed (since $S$ is closed) and maximal scattering dissipative (since $S$ is maximal scattering dissipative). Thus, by Theorem 2.5, $S_{P}$ is a scattering passive system node.

\section{REFERENCES}

[AN96] Damir Z. Arov and Mark A. Nudelman, Passive linear stationary dynamical scattering systems with continuous time, Integral Equations Operator Theory 24 (1996), 1-45. MR.1366539(96k:47016)

[MS06] Jarmo Malinen and Olof J. Staffans, Conservative boundary control systems, J. Differential Equations 231 (2006), 290-312. MR2287888 (2008a:93010)

[MSW06] Jarmo Malinen, Olof J. Staffans, and George Weiss, When is a linear system conservative?, Quart. Appl. Math. 64 (2006), 61-91. MR2211378 (2007m:47022)

[Phi59] Ralph S. Phillips, Dissipative operators and hyperbolic systems of partial differential equations, Trans. Amer. Math. Soc. 90 (1959), 193-254. MR0104919 (21:3669) 
[Sta01] Olof J. Staffans, J-energy preserving well-posed linear systems, Internat. J. Appl. Math. Comput. Sci. 11 (2001), 1361-1378. MR1885509 (2002k:93045)

[Sta02a] , Passive and conservative continuous-time impedance and scattering systems. Part I: Well-posed systems, Math. Control Signals Systems 15 (2002), 291-315. MR.1942089(2003i:93024)

[Sta02b] , Passive and conservative infinite-dimensional impedance and scattering systems (from a personal point of view), Mathematical Systems Theory in Biology, Communication, Computation, and Finance (New York), IMA Volumes in Mathematics and its Applications, vol. 134, Springer-Verlag, 2002, pp. 375-414. MR2043247 (2004m:93072)

[Sta05] Well-posed linear systems, Cambridge University Press, Cambridge and New York, 2005. MR2154892(2006k:93003)

[SW10] Roland Schnaubelt and George Weiss, Two classes of passive time-varying well-posed linear systems, Math. Control Signals Systems 21 (2010), no. 4, 265-301. MR2672788 (2011h:93018)

[SW12] Olof J. Staffans and George Weiss, A physically motivated class of scattering passive linear systems, submitted to SIAM J. Control Optim., http://users.abo.fi/staffans, 2012.

[WST01] George Weiss, Olof J. Staffans, and Marius Tucsnak, Well-posed linear systems - a survey with emphasis on conservative systems, Internat. J. Appl. Math. Comput. Sci. 11 (2001), 7-34. MR1835146 (2002f:93068)

Department of Mathematics, Åbo Akademi University, Fin-20500 Åbo, Finland $U R L:$ http://users .abo.fi/staffans/ 